\title{
Analysis of Secondary Leukemia and Myelodysplastic Syndrome After Chemotherapy for Solid Organ Tumors Using the Food and Drug Administration Adverse Event Reporting System (FAERS)
}

\author{
Takehiro Kawashiri ${ }^{1}$, Daisuke Kobayashi ${ }^{1}$, Mayako Uchida ${ }^{2}$, Shiori Hiromoto ${ }^{1}$, Masashi Inoue ${ }^{1}$, Hajime Ikeda ${ }^{1}$, Mizuki \\ Inoue $^{1}$, Takao Shimazoe ${ }^{1}$ \\ ${ }^{1}$ Department of Clinical Pharmacy and Pharmaceutical Care, Graduate School of Pharmaceutical Sciences, Kyushu \\ University, Fukuoka, Japan; ${ }^{2}$ Education and Research Center for Clinical Pharmacy, Osaka University of Pharmaceutical \\ Sciences, Osaka, Japan.
}

Corresponding author: Takehiro Kawashiri, Department of Clinical Pharmacy and Pharmaceutical Care, Graduate School of Pharmaceutical Sciences, Kyushu University, 3-1-1 Maidashi, Higashi-ku, Fukuoka 812-8582, Japan; TEL: (+81) 92 642 6573; Fax: (+81) 92642 6647; email: tkawa@med.kyushu-u.ac.jp

Received September 8, 2021, Revised, September 8, 2021, Accepted, October 8, 2021, Published, October 12, 2021

\begin{abstract}
Purpose: As the prognosis of cancer patients deteriorates, secondary carcinogenesis after chemotherapy, especially secondary hematological malignancies, becomes a serious problem. However, information on the frequency and time of onset of secondary hematological malignancies and the risk of hematological malignancy with different drugs is scarce. This study aimed to evaluate the incidence of leukemia and myelodysplastic syndrome in patients with solid tumors, including breast, colon, gastric, pancreatic, small cell lung, non-small cell lung, esophageal, ovarian, cervical, and endometrial cancers. Methods: Using the United States Food and Drug Administration Adverse Event Reporting System, we analyzed the reporting rates, reporting odds ratios, and the reporting onset times of secondary leukemia and myelodysplastic syndrome for each drug used. Results: The leukemia reporting rates were higher in breast, small cell lung, ovarian, and endometrial cancers than in other cancers, and the myelodysplastic syndrome reporting rates were higher in ovarian and endometrial cancers than in other cancers. For each cancer type, the reporting odds ratios of cytocidal anticancer agents, such as taxanes, anthracyclines, alkylating agents, platinum, and topoisomerase inhibitors, were higher than those of other drugs. Alternatively, the reporting odds ratios of molecular targeted drugs and immune checkpoint inhibitors were not higher than those of other drugs. Approximately half of the cases of leukemia and myelodysplastic syndrome were reported within 1 to 4 years after chemotherapy. Conclusions: Our study clarified the risks of leukemia and myelodysplastic syndrome for several anticancer drugs in patients with solid tumors. Our data may aid in the assessment of the risks of secondary leukemia and myelodysplastic syndrome when medical oncologists, clinical pharmacists, and patients select chemotherapy regimens.
\end{abstract}

\section{INTRODUCTION}

Although the development of chemotherapy has prolonged the life expectancy of cancer patients, secondary cancers, especially therapy-related myeloid neoplasms (t-MNs), are a serious problem $(1,2)$. Many reports have indicated that breast cancer patients are at a high risk of marrow neoplasms after chemotherapy (3-5) and that treatment for Hodgkin's lymphoma is a risk factor for leukemia (6). However, these reports were limited to cancer type and specific anticancer drugs, and there exists little comprehensive information on the extent to which each anticancer drug increased the risk for t-MN, including leukemia and myelodysplastic syndrome (MDS), in each cancer type. Therefore, medical oncologists, clinical pharmacists, and patients have not been able to adequately consider the risk of t-MN when selecting chemotherapy regimens.

Large-scale health information databases are beginning to be used in drug discovery and development. The US Food and Drug Administration Adverse Event Reporting System (FAERS) has registered more than three million spontaneous reports of adverse events (7) and is an effective tool for comprehensive risk assessments of adverse drug events.

In this study, we successfully conducted a comprehensive survey and analyzed the reporting frequency and the time to the onset of leukemia and MDS and the risk of leukemia and MDS for different drugs in patients with different solid tumors using 
reports from FAERS.

ABBREVIATION. t-MN: therapy-related myeloid neoplasm; MDS: myelodysplastic syndrome; FAERS: US Food and Drug Administration Adverse Event Reporting System; AML: acute myeloid leukemia; ROR: reporting odds ratio; CI: confidence interval

\section{METHODS}

Of the 11,289,189 adverse event reports from FAERS from 1997 to the second quarter of 2019 , cases of breast (77,096 reports), colon (11,499 reports), gastric $(6,866$ reports), pancreatic (12,368 reports), small cell lung (3,625 reports), non-small cell lung (36,930 reports), esophageal (3,409 reports), ovarian $(19,402$ reports), cervical (1,818 reports), and endometrial (1,319 reports) cancers were included in this study. The report data were extracted using CzeekV Pro (version 5.0.12, INTAGE Healthcare Inc., Tokyo, Japan, accessed September 2019). Reports of adverse events containing the word "leukemia" were considered leukemia reports; thus, the leukemia reports included acute lymphoblastic leukemia, chronic myeloid leukemia, chronic lymphoblastic leukemia, and acute myeloid leukemia (AML). We investigated the reporting rates, reporting odds ratios (RORs), and the times to onset of leukemia and MDS reported as adverse events after anticancer drug use in the reports of each cancer type. The RORs and 95\% confidence intervals (CIs) were calculated using Eq. (8).

$$
\begin{gathered}
\operatorname{ROR}=\frac{\mathrm{n} 11 / \mathrm{n} 21}{\mathrm{n} 12 / \mathrm{n} 22} \\
95 \% \mathrm{CI}=\exp \left[\log (\mathrm{ROR}) \pm 1.96 \sqrt{\frac{1}{\mathrm{n} 11}+\frac{1}{\mathrm{n} 12}+\frac{1}{\mathrm{n} 21}+\frac{1}{\mathrm{n} 22}}\right] \text { (2) }
\end{gathered}
$$

In these formulas, $\mathrm{n} 11$ refers to patients who used an anticancer drug and reported a hematological malignancy, such as leukemia or MDS; n12 refers to patients who used an anticancer drug but did not report a hematological malignancy; $\mathrm{n} 21$ refers to patients who did not use an anticancer drug but reported a hematological malignancy; and $\mathrm{n} 22$ refers to patients who did not use an anticancer drug and did not report a hematological malignancy. We excluded reports without information regarding the onset time of leukemia or MDS from the analysis. Statistical analysis was performed using a chi-square test (StatView; Abacus Concepts, Berkeley, California, USA). Statistical significance was set at $P<0.05$. The data that support the findings of this study are available from the corresponding author upon reasonable request.

\section{RESULTS}

\section{Reporting rates of leukemia and MDS}

Reports of leukemia accounted for $0.07-1.06 \%$ of all reports of adverse effects for each cancer type (Figure 1A). The reporting rates of leukemia were high in endometrial (1.06\%), small cell lung (1.02\%), ovarian $(1.02 \%)$, and breast $(0.90 \%)$ cancers. Conversely, there are few reports of leukemia in colorectal $(0.07 \%)$, pancreatic $(0.11 \%)$, and nonsmall cell lung $(0.12 \%)$ cancers.

Reports of MDS accounted for $0.08-0.83 \%$ of all reports of adverse effects for each cancer type (Figure 1B). The reporting rates of MDS were high in endometrial $(0.83 \%)$ and ovarian $(0.62 \%)$ cancers. Conversely, there are few reports of MDS in colorectal $(0.06 \%)$, pancreatic $(0.08 \%)$, gastric $(0.10 \%)$, and non-small cell lung $(0.11 \%)$ cancers.

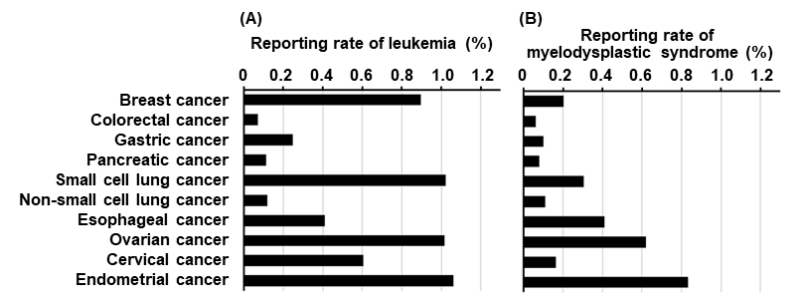

Figure 1. Reporting rates of leukemia (A) and myelodysplastic syndrome (MDS) (B).

\section{RORs of leukemia in breast cancer}

The RORs for docetaxel, paclitaxel, doxorubicin, epirubicin, cyclophosphamide, fluorouracil, methotrexate, and tamoxifen were greater than one (Figure 2). For patients on cyclophosphamide, fluorouracil, methotrexate, and doxorubicin, the RORs (95\% CIs) were 11.89 (10.09-13.96), 10.48 (8.97-12.25), 9.62 (7.56-12.24), and 6.11 (5.21$7.16)$, respectively, and the reporting rates were $3.81 \%, 5.75 \%, 7.23 \%$, and $3.76 \%$, respectively. The RORs for capecitabine, exemestane, fulvestrant, trastuzumab, lapatinib, pertuzumab, trastuzumab emtansine, neratinib, palbociclib, and bevacizumab were less than one.

\section{RORs of leukemia in small cell lung cancer}

The ROR $(95 \% \mathrm{CI})$ of etoposide, a topoisomerase inhibitor, was 3.68 (1.73-7.83), and the reporting rate in patients on etoposide was $1.68 \%$ (Figure 3 ). There were no reports of leukemia in patients receiving nivolumab or ipilimumab, which are immune checkpoint inhibitors $(P<0.05)$. 


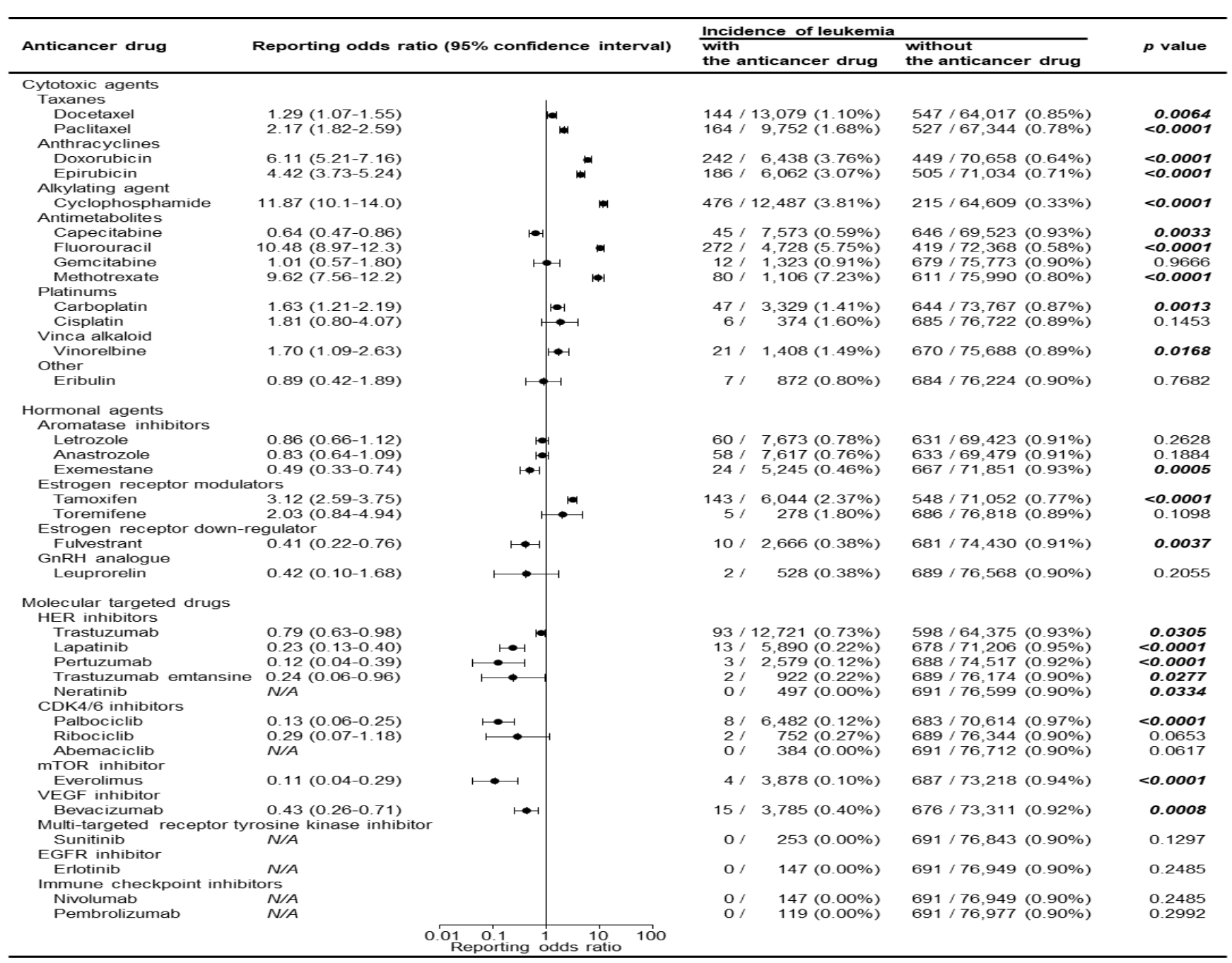

Figure 2. Reporting odds ratios for leukemia in breast cancer. GnRH: gonadotropin releasing hormone, HER: human epidermal growth factor receptor, CDK4/6: cyclin-dependent kinase 4/6, mTOR: mammalian target of rapamycin, VEGF: vascular endothelial growth factor, EGFR: epidermal growth factor receptor.

\section{RORs of leukemia and MDS in ovarian cancer}

For leukemia, the RORs for carboplatin, cisplatin, paclitaxel, docetaxel, doxorubicin, gemcitabine, etoposide, irinotecan, cyclophosphamide, trabectedin, and olaparib were greater than one (Figure 4). In patients receiving cyclophosphamide, etoposide, and irinotecan, the RORs $(95 \% \mathrm{CI})$ were 11.40 (7.76-16.75), 11.17 (6.96-17.93), and 10.87 (5.58-21.20), respectively, and the reporting rates were $8.97 \%, 9.38 \%$, and $9.62 \%$, respectively. The RORs of niraparib and rucaparib were less than one.

For MDS, the RORs for carboplatin, cisplatin, paclitaxel, docetaxel, doxorubicin, etoposide, irinotecan, cyclophosphamide, and olaparib were greater than one (Figure 5). In patients on irinotecan and cyclophosphamide, the RORs $(95 \% \mathrm{CI})$ were $18.56(9.42-36.57)$ and $11.21 \quad(6.92-18.16)$, respectively, and the reporting rates were $9.62 \%$ and
$5.54 \%$, respectively. The RORs of bevacizumab, niraparib, and rucaparib were less than one.

\section{RORs of leukemia and MDS in endometrial cancer}

Carboplatin showed high RORs for both leukemia and MDS (Figures 6 and 7).

RORs of leukemia and MDS in other solid tumors The RORs of leukemia and MDS in other solid tumors are shown in the supporting information (Supplimentary Figures S1-S14).

\section{Reporting onset times of leukemia and MDS}

The onset times of leukemia and MDS were extracted from the reports of patients with breast, colorectal, gastric, pancreatic, small cell lung, non-small cell lung, esophageal, ovarian, cervical, and endometrial 


\begin{tabular}{|c|c|c|c|c|}
\hline \multirow[b]{2}{*}{ Anticancer drug } & \multirow[b]{2}{*}{ Reporting odds ratio ( $95 \%$ confidence interval) } & \multicolumn{2}{|l|}{ Incidence of leukemia } & \multirow[b]{2}{*}{$p$ value } \\
\hline & & $\begin{array}{l}\text { with } \\
\text { the anticancer drug }\end{array}$ & $\begin{array}{l}\text { without } \\
\text { the anticancer drug }\end{array}$ & \\
\hline \multicolumn{5}{|c|}{ Cytotoxic agents } \\
\hline \multicolumn{5}{|c|}{ Topoisomerase inhibitors } \\
\hline Etoposide & $3.68(1.73-7.83)$ & $28 / 1,671(1.68 \%)$ & $9 / 1,954(0.46 \%)$ & 0.0003 \\
\hline Irinotecan & $1.10(0.46-2.66)$ & $541(1.11 \%)$ & $31 / 3,084(1.01 \%)$ & 0.8245 \\
\hline Topotecan & $0.24(0.03-1.74)$ & $376(0.27 \%)$ & $36 / 3,249(1.11 \%)$ & 0.1241 \\
\hline \multicolumn{5}{|l|}{ Platinums } \\
\hline Carboplatin & $1.41(0.72-2.74)$ & $14 / 1,098(1.28 \%)$ & $23 / 2,527(0.91 \%)$ & 0.3152 \\
\hline Cisplatin & $0.99(0.49-2.00)$ & $11 / 1,088(1.01 \%)$ & $26 / 2,537(1.02 \%)$ & 0.9698 \\
\hline \multicolumn{5}{|l|}{ Taxane } \\
\hline Paclitaxel & $N / A$ & $145(0.00 \%)$ & $37 / 3,480(1.06 \%)$ & 0.2120 \\
\hline \multicolumn{5}{|c|}{$\begin{array}{l}\text { Molecular targeted drugs } \\
\text { Immune checkpoint inhibitors }\end{array}$} \\
\hline Nivolumab & $N / A$ & $468(0.00 \%)$ & $37 / 3,157(1.17 \%)$ & 0.0186 \\
\hline \multirow[t]{2}{*}{ Ipilimumab } & $N / A$ & $358(0.00 \%)$ & $37 / 3,267(1.13 \%)$ & 0.0430 \\
\hline & $0.01 \begin{array}{llll}0.1 & 1 & 10 & 100 \\
\text { Reporting } & \text { odds } & \text { ratio } & \end{array}$ & & & \\
\hline
\end{tabular}

Figure 3. Reporting odds ratios of leukemia in small cell lung cancer.

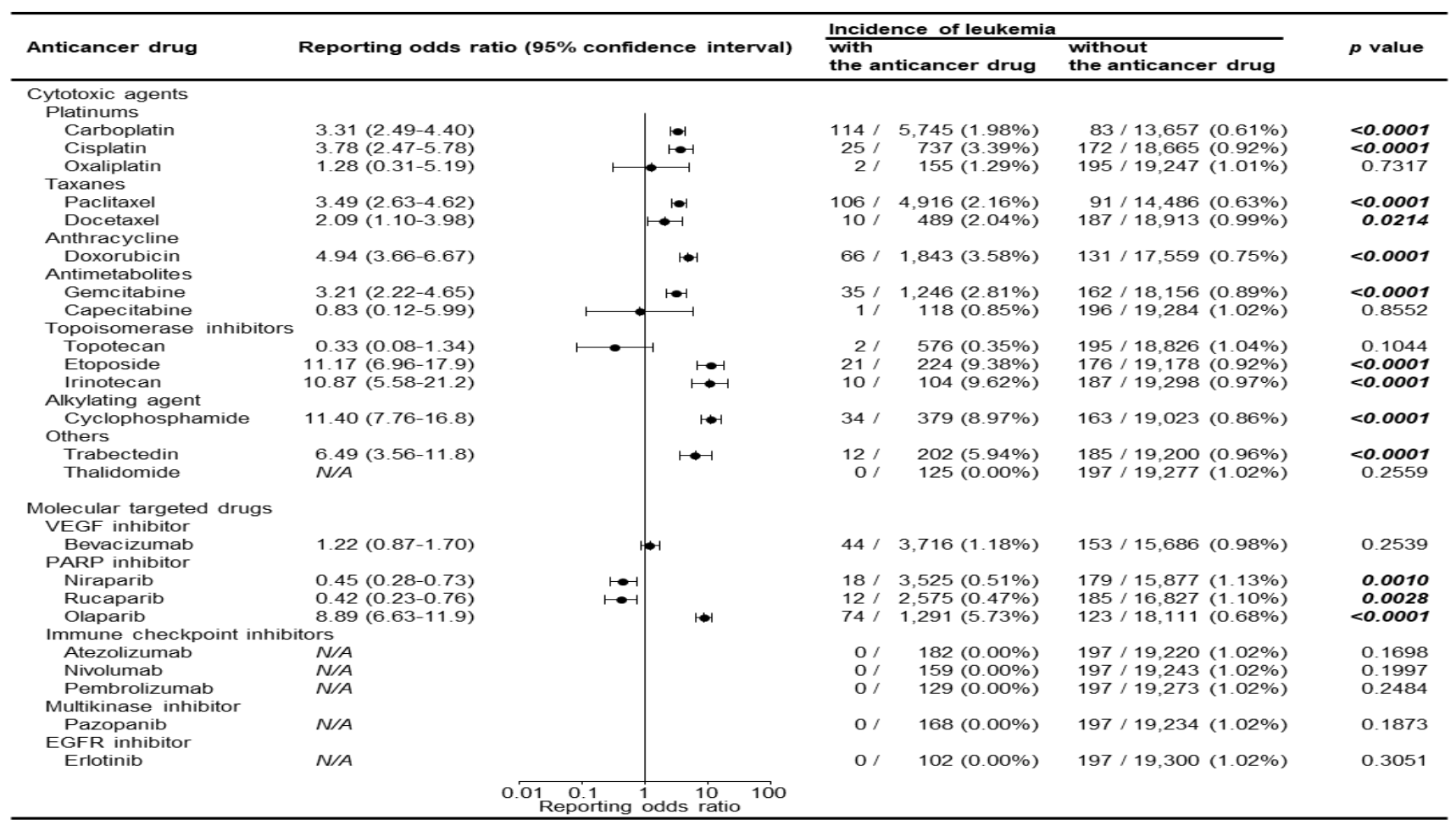

Figure 4. Reporting odds ratios of leukemia in ovarian cancer. VEGF: vascular endothelial growth factor, PARP: poly (ADPribose) polymerase, EGFR: epidermal growth factor receptor.

cancers. The reporting onset times of leukemia and MDS for each anticancer drug are shown in Figure 8. The median reporting onset times were 1.08-2.35 and 0.99-3.41 years for leukemia and MDS, respectively. Some cases of leukemia and MDS have been reported for over 20 years after chemotherapy. Approximately half of the cases of leukemia and MDS have been reported 1-4 years after chemotherapy. The results of the anticancer drugs that significantly increased the risk of leukemia and MDS are shown in Figures 2-7. Reports without information regarding the onset time were excluded.

\section{DISCUSSION}

Leukemia and MDS accounted for $0.07-1.06 \%$ and $0.08-0.83 \%$ of adverse event reports in FAERS, respectively, for each solid cancer in our study. These reporting rates do not refer to the incidence rates after the use of an anticancer drug because they were calculated in this study by dividing the number of leukemia and MDS reports by the total number of adverse event reports. However, these results demonstrate that a considerable number of patients develop secondary leukemia and MDS. 


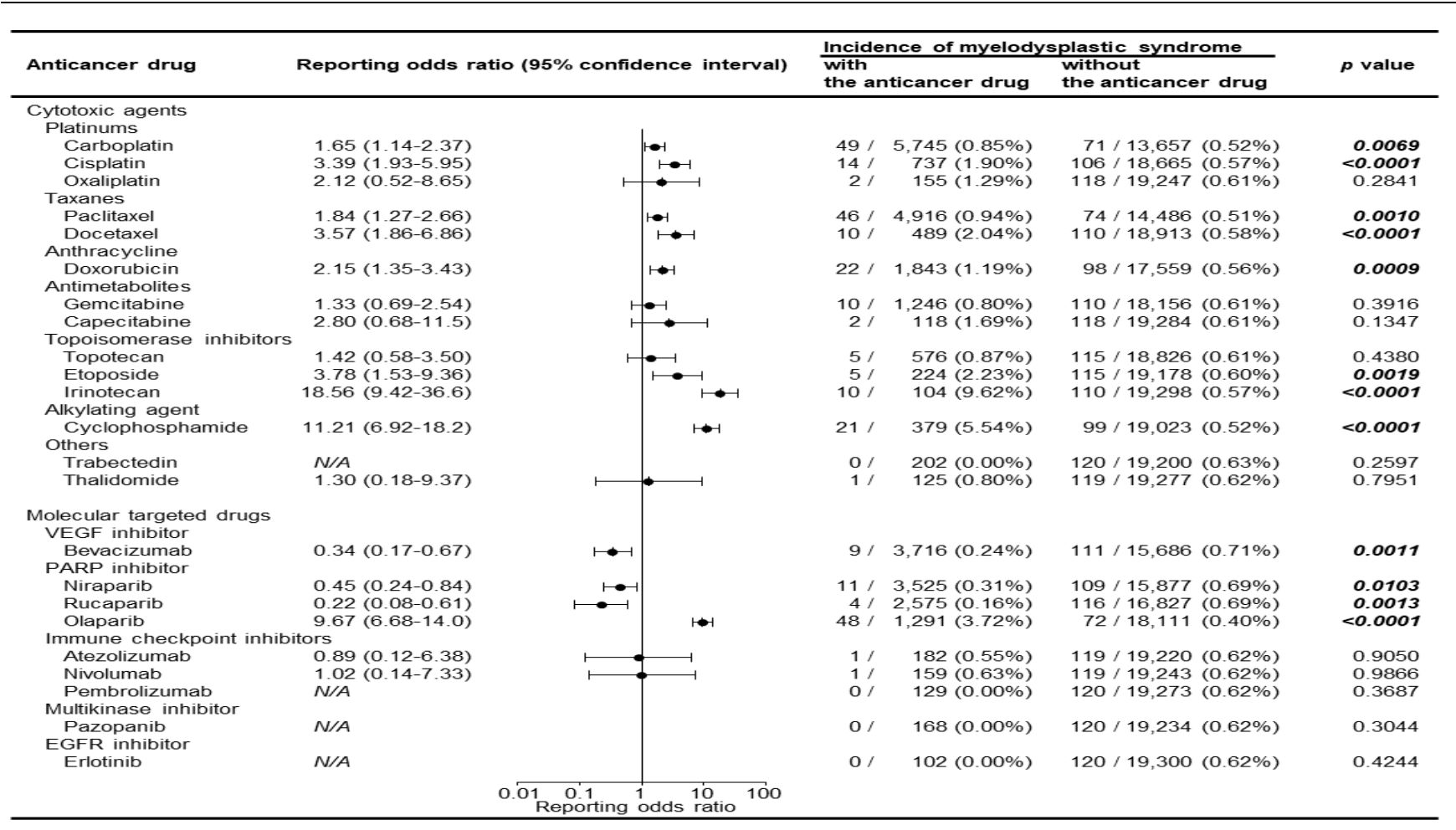

Figure 5. Reporting odds ratios of myelodysplastic syndrome (MDS) in ovarian cancer. VEGF: vascular endothelial growth factor, PARP: poly (ADP-ribose) polymerase, EGFR: epidermal growth factor receptor.

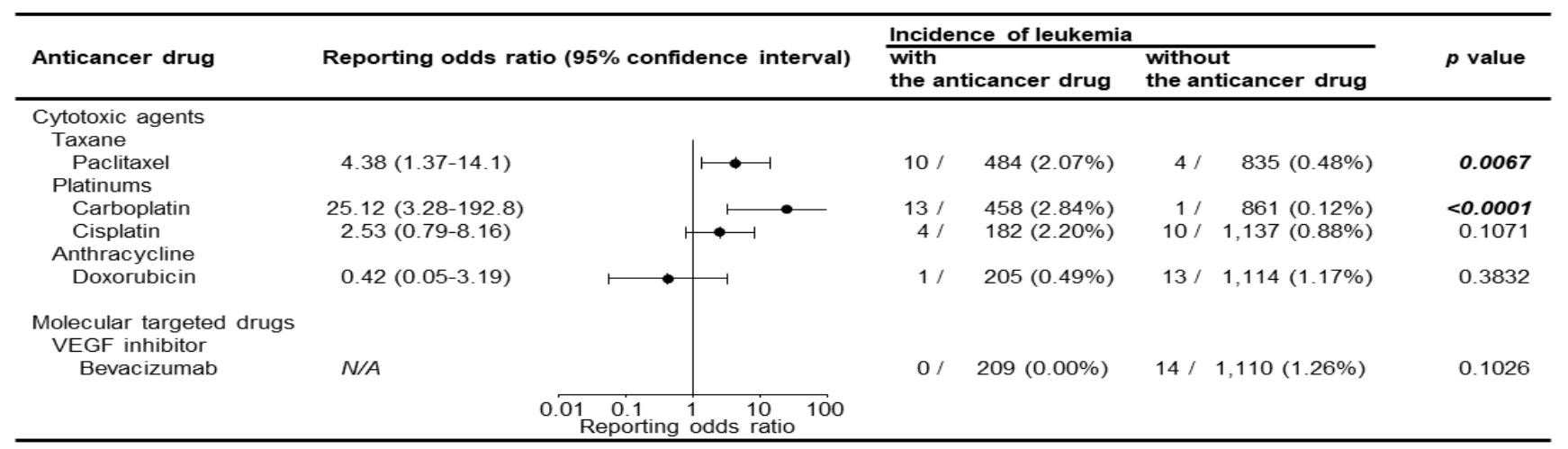

Figure 6. Reporting odds ratios of leukemia in endometrial cancer. VEGF: vascular endothelial growth factor.

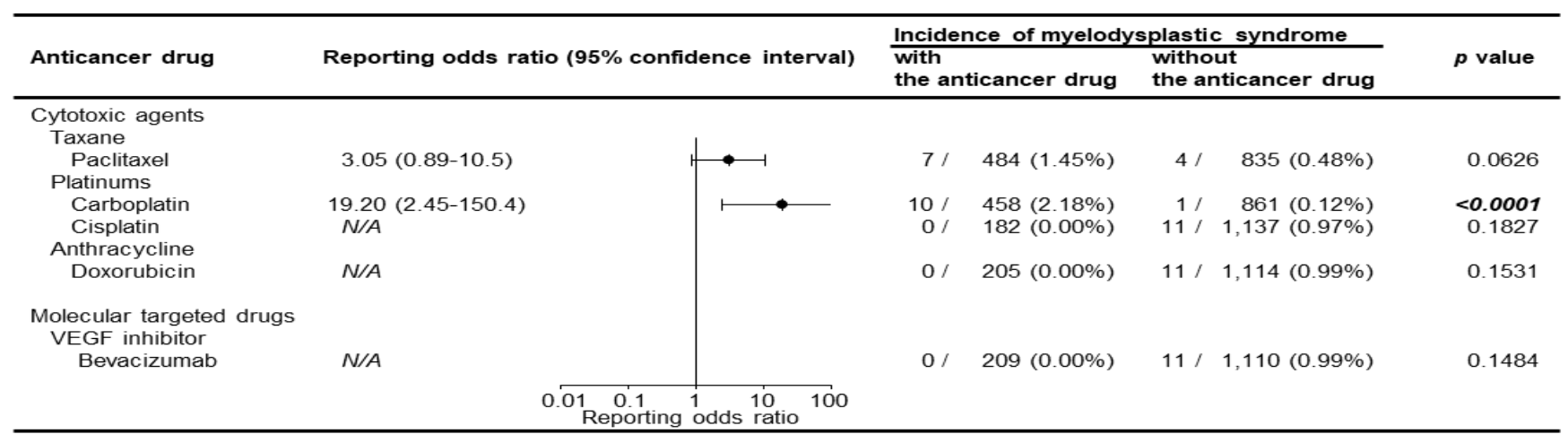

Figure 7. Reporting odds ratios of myelodysplastic syndrome (MDS) in endometrial cancer. VEGF: vascular endothelial growth factor. 

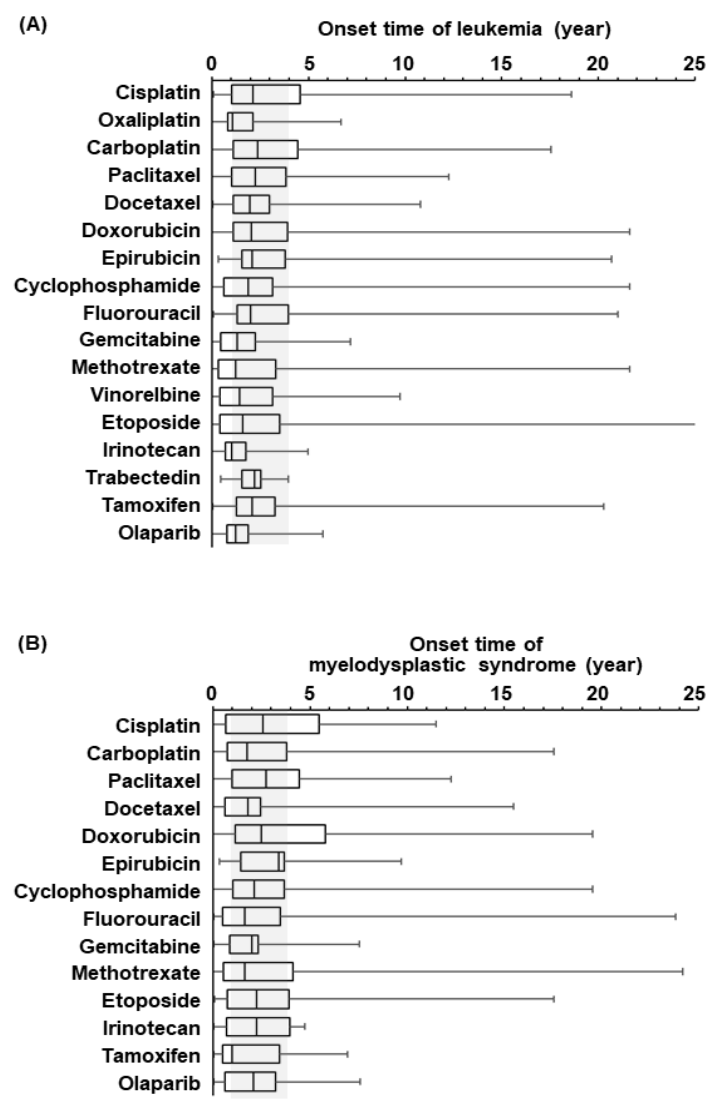

Figure 8. Reporting onset times of leukemia (A) and myelodysplastic syndrome (MDS) (B).

When analyzed by cancer type, the reporting rates of leukemia were high in breast, small cell lung, ovarian, and endometrial cancers, and low in colorectal, pancreatic, and non-small cell lung cancers. One factor that affects the difference in reporting rates among cancer types is the difference in life expectancy for each cancer. For example, leukemia was more likely to be reported in breast cancer patients because breast cancer has a good prognosis, and the opposite is true for pancreatic cancer. Another factor that affects reporting rates is the difference in the type of anticancer drug used.

Many previous reports have indicated secondary malignancies in breast cancer patients (912). In our study, patients receiving cytocidal anticancer drugs, including taxanes (paclitaxel and docetaxel), anthracyclines (doxorubicin and epirubicin), alkylating agents (cyclophosphamide), and antimetabolites (fluorouracil and methotrexate), had significantly higher reported rates of leukemia in the reports of breast cancer. These drugs are widely used as adjuvant chemotherapy for breast cancer (13-15). Thus, medical oncologists, clinical pharmacists, and patients should fully understand and consider the risks of secondary leukemia when initiating adjuvant therapy for breast cancer. Taxanes, anthracyclines, alkylating agents, and antimetabolites showed high RORs for leukemia in cancer types other than breast cancer. Several genetic abnormalities have been reported in patients with therapy-related AML and MDS (16). AML after chemotherapy with anthracyclines, which are DNAtopoisomerase II inhibitors, is often associated with chromosomal translocations involving chromosome bands 11q23 (MLL) and 21q22 (RUNX1) (17,18). Monosomy 5/deletion 5q, monosomy 7/deletion 7q, or both, are found in patients with AML after alkylating agents (18). These effects on the chromosome are considered to be the major mechanisms of $\mathrm{t}-\mathrm{MN}$ induced by cytocidal anticancer drugs. In addition, patients on molecular targeted agents, including HER inhibitors (trastuzumab, lapatinib, pertuzumab, trastuzumab emtansine, and neratinib), cyclin-dependent kinase (CDK) 4/6 inhibitors (palbociclib), and vascular endothelial growth factor (VEGF) inhibitors (bevacizumab), had significantly lower reported rates of leukemia in the reports of breast cancer in this study. These results may be because molecular targeted drugs are less likely to cause chromosomal abnormalities than cytotoxic anticancer drugs. Another reason could be that CDK4/6 inhibitors, VEGF inhibitors, and trastuzumab emtansine are often used for advanced breast cancer (19-21) rather than adjuvant chemotherapy. Regardless, it can be concluded that t-MN after molecular-targeted chemotherapy is less of a problem in breast cancer patients. Regarding hormonal agents, only tamoxifen was associated with a high risk of leukemia. Tamoxifen was previously reported to be a risk factor for endometrial cancer (22). Several mechanisms, such as estrogenic effects, the mammalian target of the rapamycin (mTOR) autophagy signaling pathway, DNA damage, and effects on driver genes, are involved in the induction of endometrial cancer by tamoxifen (23). Some of these mechanisms may also be involved in the development of t-MN. Because many patients take tamoxifen, patients and medical teams need to be aware of the risks of t-MN.

Among cancer types other than breast cancer, there was a tendency for cytocidal drugs to increase the risk of leukemia, whereas molecular targeted drugs had a low risk. Platinum showed high RORs in colorectal (Supplementary Figure S2), gastric (Supplementary Figure S4), non-small cell lung (Supplementary Figure S9), ovarian, cervical (Supplementary Figure S13), and endometrial 
cancers, and topoisomerase inhibitors showed high RORs in small cell lung, non-small cell lung (Supplementary Figure S9), and ovarian cancers. Previous studies have reported that the use of cisplatin, a platinum drug, approximately triples the risk of leukemia in ovarian and testicular cancer (24, 25). Cytocidal anticancer drugs could be considered a risk factor for t-MN in almost all types of cancer. In addition, there have been few reports of leukemia in patients treated with immune checkpoint inhibitors. Considering the mechanism of action, the risk of t$\mathrm{MN}$ due to immune checkpoint inhibitors is considered low. However, since the use of immune checkpoint inhibitors has recently increased, it is necessary to continue collecting information. In this study, we analyzed both leukemia and MDS, and found a correlation between the risk of leukemia and MDS for each drug and cancer type.

Approximately half of the cases of leukemia and MDS were reported 1-4 years after chemotherapy in our analysis. Some cases of leukemia and MDS have been reported for over 20 years after chemotherapy. A previous report also indicated the risk of secondary cancer up to 40 years after treatment in patients with Hodgkin's lymphoma (26). Since t-MN is a long-term adverse event, it must be considered when initiating chemotherapy in children, adolescents, young adults, and other young people.

Since t-MN occurs less frequently than other adverse effects associated with chemotherapy, including nausea, vomiting, and bone marrow suppression, it is easily neglected when chemotherapy is initiated. Other adverse effects, such as nausea, vomiting, and neutropenia, can be treated with supportive care; however, it can often be fatal or challenging. Thus, medical oncologists, clinical pharmacists, and patients should consider the t-MN risk of each drug in each cancer type when selecting chemotherapy regimens. There have been many studies on t-MN since early times $(9-12,22,24,25,27-$ 32). Most of these studies were limited to cancer types and causative agents, and had a small number of cases (9-12,22,24,25,27-30). Recently, large-scale studies on secondary cancers have been reported (3337). Chaturvedi AK. and colleagues studied the risk of second cancers in cervical cancer with radiation treatment, using data from 104,760 one-year survivors of cervical cancer reported to 13 population-based cancer registries in Denmark, Finland, Norway, Sweden, and the United States (33). They reported that cervical cancer patients treated with radiotherapy are at an increased risk of second cancers beyond 40 years of follow-up (33). Moreover, Ju HY. and colleagues reported that childhood cancer survivors were at a 20 -fold higher risk of developing a malignant neoplasm compared to the general population, through a registry-based study of 5.6 years of follow-up using the medical data from the Korea Central Cancer Registry $(28,405$ patients) (36). Furthermore, a study by Morton LM. and colleagues assessed the tMDS/AML risk after chemotherapy for solid cancer using cancer registries from the Surveillance, Epidemiology, and End Results Program and Medicare claims (700,612 patients) (37). These large studies take a great deal of time and effort and therefore have a large number of cases and reliable data. However, these studies are limited in terms of cancer type and other conditions, and there is a lack of data assessing which drugs are associated with a higher risk. Our study assessed the risk of t-MN with each drug in each cancer type, which has not been assessed in previous studies. Although there are some problems with adverse event reporting databases, such as data heterogeneity, they are useful tools for analyzing large-scale and comprehensive information. Our data may not directly reflect the risks associated with the drugs themselves. However, these data seem to reflect cases that are based on actual reports (i.e., cases that may present problems in clinical practice). The results of our analysis using a large adverse event database may compensate for the lack of previous studies on the t-MN risk of each drug in each cancer.

\section{CONCLUSION}

This study clarified the risks of t-MN for several anticancer drugs in patients with different solid tumors. The data presented here and in the supporting information (Supplementary Figures S1-S14) could be useful for assessing the risks of secondary leukemia and MDS when medical oncologists, clinical pharmacists, and patients select chemotherapy regimens.

ACKNOWLEDGMENT. The authors thank Editage (www.editage.jp) for the English language review.

CONFLICT OF INTEREST STATEMENT. The authors declare no conflicts of interest associated with this manuscript. 


\section{REFERENCES}

1. Fianchi L, Criscuolo M, Fabiani E, Falconi G, Maraglino AME, Voso MT et al. Therapyrelated myeloid neoplasms: clinical perspectives. Onco Targets Ther. 2018; 11:5909-5915. doi: 10.2147/OTT.S101333.

2. Demoor-Goldschmidt C, de Vathaire F. Review of risk factors of secondary cancers among cancer survivors. Br J Radiol. 2019; 92:20180390. doi: 10.1259/bjr.20180390.

3. Le Deley MC, Suzan F, Cutuli B, Delaloge S, Shamsaldin A, Linassier $\mathrm{C}$ et al. Anthracyclines, mitoxantrone, radiotherapy, and granulocyte colony-stimulating factor: risk factors for leukemia and myelodysplastic syndrome after breast cancer. J Clin Oncol. 2007; 25:292-300. doi:10.1200/JCO.2006.05.9048.

4. Wolff AC, Blackford AL, Visvanathan K, Rugo HS, Moy B, Goldstein LJ et al. Risk of marrow neoplasms after adjuvant breast cancer therapy: the national comprehensive cancer network experience. J Clin Oncol. 2015; 33:340-348. doi:10.1200/JCO.2013.54.6119.

5. Jabagi MJ, Goncalves A, Vey N, Le Tri T, Zureik M, Dray-Spira R. Risk of hematologic malignant neoplasms after postoperative treatment of breast cancer. Cancers (Basel). 2019; 11:1463. doi:10.3390/cancers11101463.

6. Schonfeld SJ, Gilbert ES, Dores GM, Lynch CF, Hodgson DC, Hall P et al. Acute myeloid leukemia following Hodgkin lymphoma: a population-based study of 35,511 patients. J Natl Cancer Inst. 2006; 98:215-218. doi:10.1093/jnci/djj017.

7. Harpaz R, DuMouchel W, LePendu P, BauerMehren A, Ryan P, Shah NH. Performance of pharmacovigilance signal-detection algorithms for the FDA adverse event reporting system. Clin Pharmacol Ther. 2013; 93:539-546. doi:10.1038/clpt.2013.24.

8. Suzuki Y, Suzuki H, Umetsu R, Uranishi H, Abe J, Nishibata $Y$ et al. Analysis of the interaction between clopidogrel, aspirin, and proton pump Inhibitors using the FDA Adverse Event Reporting System Database. Biol Pharm Bull. 2015; 38:680-686. doi:10.1248/bpb.b14-00191.

9. Chaplain G, Milan C, Sgro C, Carli PM,
Bonithon-Kopp C. Increased risk of acute leukemia after adjuvant chemotherapy for breast cancer: a population-based study. J Clin Oncol. 2000; 18:2836-2842. doi:10.1200/JCO.2000.18.15.2836.

10. Martin MG, Welch JS, Luo J, Ellis MJ, Graubert TA, Walter MJ. Therapy related acute myeloid leukemia in breast cancer survivors, a population-based study. Breast Cancer Res Treat. 2009; 118:593-598. doi:10.1007/s10549-009-0376-3.

11. Molina-Montes E, Requena M, SánchezCantalejo E, Fernández MF, Arroyo-Morales $\mathrm{M}$, Espín J et al. Risk of second cancers cancer after a first primary breast cancer: a systematic review and meta-analysis. Gynecol Oncol. 2015; 136:158-171. doi:10.1016/j.ygyno.2014.10.029.

12. Wei JL, Jiang YZ, Shao ZM. Survival and chemotherapy-related risk of second primary malignancy in breast cancer patients: a SEERbased study. Int J Clin Oncol. 2019; 24:934940. doi:10.1007/s10147-019-01430-0.

13. Bonadonna G, Valagussa $\mathrm{P}$, Moliterni A, Zambetti M, Brambilla C. Adjuvant cyclophosphamide, methotrexate, and fluorouracil in node-positive breast cancer: the results of 20 years of follow-up. N Engl J Med. 1995; 332:901-906. doi:10.1056/NEJM199504063321401.

14. De Laurentiis M, Cancello G, D'Agostino D, Giuliano M, Giordano A, Montagna E et al. Taxane-based combinations as adjuvant chemotherapy of early breast cancer: a metaanalysis of randomized trials. J Clin Oncol. 2008;

26:44-53. doi:10.1200/JCO.2007.11.3787.

15. Early Breast Cancer Trialists' Collaborative Group (EBCTCG), Peto R, Davies C, Godwin J, Gray R, Pan HC et al. Comparisons between different polychemotherapy regimens for early breast cancer: meta-analyses of long-term outcome among 100,000 women in 123 randomised trials. Lancet. 2012; 379:432-444. doi:10.1016/S0140-6736(11)61625-5.

16. Qian Z, Joslin JM, Tennant TR, Reshmi SC, Young DJ, Stoddart A et al. Cytogenetic and genetic pathways in therapy-related acute myeloid leukemia. Chem Biol Interact. 2010; 184:50-57. doi:10.1016/j.cbi.2009.11.025.

17. Athanasiadou A, Saloum R, Zorbas I, 
Tsompanakou A, Batsis I, Fassas A et al. Therapy-related myelodysplastic syndrome with monosomy 5 and 7 following successful therapy for acute promyelocytic leukemia with anthracyclines. Leuk Lymphoma. 2002; 43:2409-2411.

doi:10.1080/1042819021000040143.

18. McNerney ME, Godley LA, Le Beau MM. Therapy-related myeloid neoplasms: when genetics and environment collide. Nat Rev Cancer. 2017; 17:513-527. doi:10.1038/nrc.2017.60.

19. Miller K, Wang M, Gralow J, Dickler M, Cobleigh M, Perez EA et al. Paclitaxel plus bevacizumab versus paclitaxel alone for metastatic breast cancer. N Engl J Med. 2007; 357:2666-2676. doi:10.1056/NEJMoa072113.

20. Turner NC, Ro J, André F, Loi S, Verma S, Iwata $\mathrm{H}$ et al. Palbociclib in hormonereceptor-positive advanced breast cancer. N Engl J Med. 2015; 373:209-219. doi:10.1056/NEJMoa1505270.

21. Perez EA, Barrios C, Eiermann W, Toi M, Im $\mathrm{YH}$, Conte $\mathrm{P}$ et al. Trastuzumab emtansine with or without pertuzumab versus trastuzumab plus taxane for human epidermal growth factor receptor 2-positive, advanced breast cancer: primary results from the phase III MARIANNE study. J Clin Oncol. 2017; 35:141-148. doi:10.1200/JCO.2016.67.4887.

22. Bernstein L, Deapen D, Cerhan JR, Schwartz SM, Liff J, McGann-Maloney E et al. Tamoxifen therapy for breast cancer and endometrial cancer risk. J Natl Cancer Inst. 1999; 91:1654-1662. doi:10.1093/jnci/91.19.1654.

23. $\mathrm{Hu}$ R, Hilakivi-Clarke L, Clarke R. Molecular mechanisms of tamoxifen-associated endometrial cancer (Review). Oncol Lett. 2015; 9:1495-1501. doi:10.3892/ol.2015.2962.

24. Travis LB, Holowaty EJ, Bergfeldt K, Lynch $\mathrm{CF}$, Kohler BA, Wiklund $\mathrm{T}$ et al. Risk of leukemia after platinum-based chemotherapy for ovarian cancer. N Engl J Med. 1999; 340:351-357. doi:10.1056/NEJM199902043400504.

25. Travis LB, Andersson M, Gospodarowicz M, van Leeuwen FE, Bergfeldt K, Lynch CF et al. Treatment-associated leukemia following testicular cancer. J Natl Cancer Inst. 2000;
92:1165-1171. doi:10.1093/jnci/92.14.1165.

26. Schaapveld M, Aleman BM, van Eggermond AM, Janus CP, Krol AD, van der Maazen RW et al. Second cancer risk up to 40 years after treatment for Hodgkin's lymphoma. N Engl J Med. 2015; 373:2499-2511. doi:10.1056/NEJMoa1505949.

27. Smith MA, Rubinstein L, Cazenave L, Ungerleider RS, Maurer HM, Heyn R et al. Report of the Cancer Therapy Evaluation Program monitoring plan for secondary acute myeloid leukemia following treatment with epipodophyllotoxins. J Natl Cancer Inst. 1993; 85:554-8. doi: 10.1093/jnci/85.7.554.

28. Smith MA, Rubinstein L, Ungerleider RS. Therapy-related acute myeloid leukemia following treatment with epipodophyllotoxins: estimating the risks. Med Pediatr Oncol. 1994;23:86-98. doi:10.1002/mpo.2950230205.

29. Forrest DL, Nevill TJ, Naiman SC, Le A, Brockington DA, Barnett MJ et al. Second malignancy following high-dose therapy and autologous stem cell transplantation: incidence and risk factor analysis. Bone Marrow Transplant. 2003;32:915-23. doi:10.1038/sj.bmt.1704243.

30. Morton LM, Dores GM, Tucker MA, Kim CJ, Onel K, Gilbert ES et al. Evolving risk of therapy-related acute myeloid leukemia following cancer chemotherapy among adults in the United States, 1975-2008. Blood. 2013; 121:2996-3004. doi:10.1182/blood-2012-08448068.

31. Pyatt DW, Aylward LL, Hays SM. Is age an independent risk factor for chemically induced acute myelogenous leukemia in children? J Toxicol Environ Health B Crit Rev. 2007;10:379-400.

doi:10.1080/15287390600975061.

32. Hijiya N, Ness KK, Ribeiro RC, Hudson MM. Acute leukemia as a secondary malignancy in children and adolescents: current findings and issues. Cancer. 2009;115:23-35. doi:10.1002/cncr.23988.

33. Chaturvedi AK, Engels EA, Gilbert ES, Chen $\mathrm{BE}$, Storm H, Lynch CF et al. Second cancers among 104,760 survivors of cervical cancer: evaluation of long-term risk. J Natl Cancer Inst. $\quad 2007$; 99:1634-1643. doi:10.1093/jnci/djm201. 
34. Granfeldt Østgård LS, Medeiros BC, Sengeløv H, Nørgaard M, Andersen MK, Dufva IH, et al. Epidemiology and clinical significance of secondary and therapy-related acute myeloid leukemia: a national population-based cohort study. J Clin Oncol. 2015; 33:3641-3649. doi:10.1200/JCO.2014.60.0890.

35. Ong DM, Farrugia H, Wei A. Therapy-related acute myeloid leukaemia and myelodysplastic syndrome in Victoria, Australia 2003-2014. Intern Med J. 2018; 48:822-829. doi:10.1111/imj.13714.

36. Ju HY, Moon EK, Lim J, Park BK, Shin HY,
Won YJ et al. Second malignant neoplasms after childhood cancer: A nationwide population-based study in Korea. PLoS One. 2018;

13:e0207243. doi:10.1371/journal.pone.0207243.

37. Morton LM, Dores GM, Schonfeld SJ, Linet MS, Sigel BS, Lam CJK et al. Association of chemotherapy for solid tumors with development of therapy-related myelodysplastic syndrome or acute myeloid leukemia in the modern era. JAMA Oncol. 2019; 5:318-325. 